OPEN ACCESS

Edited by:

Min-Cherl Jung

University of Tsukuba, Japan

Reviewed by:

Li Lei,

Sichuan University, China Shanmin Wang, University of Nevada, Las Vegas,

United States

*Correspondence:

Zhuo Yu

yuzhuo6179@163.com

Specialty section:

This article was submitted to Physical Chemistry and Chemical

Physics,

a section of the journal

Frontiers in Physics

Received: 05 June 2020

Accepted: 28 July 2020

Published: 04 September 2020

Citation:

Li H, Ma S, Chen L and Yu Z (2020)

Carbon-Deficient Titanium Carbide With Highly Enhanced Hardness.

Front. Phys. 8:364

doi: 10.3389/fphy.2020.00364

\section{Carbon-Deficient Titanium Carbide With Highly Enhanced Hardness}

\author{
Hui $\mathrm{Li}^{1}$, Shuailing $\mathrm{Ma}^{2}$, Lixue Chen ${ }^{2}$ and Zhuo Yu${ }^{3 *}$ \\ ${ }^{1}$ Key Laboratory for lonospheric Observation and Simulation, School of Mathematics and Physics, Guangxi University for \\ Nationalities, Nanning, China, ${ }^{2}$ State Key Laboratory of Superhard Materials, College of Physics, Jilin University, Changchun, \\ China, ${ }^{3}$ School of Physics and Electronic Information, Baicheng Normal University, Baicheng, China
}

We report the synthesis of a polycrystalline specimen of $\mathrm{TiC}_{1-x}$ under high-pressure and high-temperature (HPHT) conditions. The carbon vacancy, crystal structure, Vicker hardness, elastic constants, and bond features of the synthesized specimen were investigated. Though the specimens were synthesized with stoichiometric ratio at high pressure, a robust carbon vacancy was observed using energy dispersive and X-ray photoelectron spectrum. $\mathrm{TiC}_{1-x}$ exhibits almost the highest asymptotic Vickers hardness in transition-metal light-element (TMLE) compounds. In this study, using Vickers hardness characterization, the asymptotic hardness was found to be $27.1 \mathrm{GPa}$. This exceeds the hardness of most transition metal borides with high boron concentrations. Based on the first-principles calculation of the Mulliken population of Ti-C bonds, the intrinsic high Vickers hardness of $\mathrm{TiC}_{1-x}$ is attributed to the combination of covalent Ti-C bonds and the optimized eight-valence-electron structure, while the extrinsic contribution comes from the harden effect of carbon defects. This work demonstrates that a higher concentration of light elements or a higher-dimensional light element framework is not the critical factor for higher hardness, and carbon vacancy is another way to strengthen the crystal structure.

Keywords: titanium carbide, crystal structure, Vicker hardness, high pressure and high temperature, carbon vacancy

\section{INTRODUCTION}

The development of multifunctional superhard materials to replace current B-C-N-based superhard materials remains challenging, and it requires the consideration of scientific, technological, and infrastructural aspects [1-5]. Transition metal carbides, borides, and nitrides exhibit high hardness, which is attributed to the strong covalent bond between the carbon and the transition metal, as well as the light element bonds [1, 5-7]. Furthermore, the transition-metal light-element compounds (TMLEs) exhibit electrical conductivity, superconductivity, and ferromagnetic as well as catalytic properties, which are useful in areas where multifunctional properties are highly desired. According to recent reports, there are many general approaches to design higher hardness materials, such as (a) having a high density of valence electrons, (b) using a high content of light element, and (c) using insulator or semiconductor materials $[8,9]$. It is generally believed that higher light-element content will lead to superhard materials because of the high percentage of covalent bonds. Using the design approaches mentioned above, many high hardness TMLEs have been designed and synthesized, including $\mathrm{FeB}_{4}, \mathrm{WB}_{4}, \mathrm{MnB}_{4}, \mathrm{MoB}_{4}, \mathrm{ZrB}_{12}$ [10-14]. Though these synthesized compounds exhibit very high hardness, their hardness values are still below the 
threshold of superhardness. Consequently, there is some doubt about whether transition-metal light-element compounds can be candidates for superhardness, or whether hybridization between TMs and LEs takes place [15]. However, recent investigation shows that some transition metal-rich counterparts, such as $\mathrm{W}_{0.5} \mathrm{Ta}_{0.5} \mathrm{~B}$, exhibit extremely high hardness [16]. This suggests that there may be some strong hybridization between transition metals and p-block light elements and there will be other new mechanisms to enhance hardness.

In the majority of transition-metal light-element compounds, TM-TM, TM-LE, and LEs-LEs bonds co-exist in the crystal structure $[9,17]$. It is difficult to determine the effect of the TM-LE bonds on hardness. Fortunately, the rock-salt structure characteristic of transition metal mono-carbides, borides, and nitrides only contain the TM-LE bonds. This is an ideal system to investigate the hybridization of TM-LE bonds in TMLEs. If higher hardness can be realized in these structures, strong hybridization can be demonstrated to exist. Binary transition metal carbides are prone to the cubic crystalline structure if the Hägg's rule $\left(R_{C} / R_{T M}<0.59\right)$ is satisfied, where $R_{C}$ refers to the atomic radius of carbon and $\mathrm{R}_{\mathrm{TM}}$ denotes the atomic radius of the transition metal atom. The ratio of carbon's atomic radius to titanium's atomic radius is 0.38 . According to previous report $[18,19]$, titanium carbide usually exhibits excellent mechanical properties, and it has been widely used in cutting and protecting applications. Thus, rock-salt structure $\mathrm{TiC}$ is an ideal system to investigate the hybridization between transition metal-d orbitals and C-2p orbitals. In addition, the use of other extrinsic strengthening effects may further enhance the hardness of titanium carbide.

In this work, the synthesis of a highly crystalline $\mathrm{TiC}$ polycrystalline specimen was performed under high pressure and high temperature conditions. The carbon concentration in this structure was researched by X-ray diffraction, energy dispersive spectroscopy, and X-ray photoelectron spectroscopy. The crystal structure, Vickers hardness, and electronic structure were characterized to determine the mechanical property. To determine the cause of its high hardness, the features of the $\mathrm{TiC}$ bonds were thoroughly investigated by both experiment and first principle calculation. The high hardness of the $\mathrm{TiC}$ specimen was attributed to the strong orbital hybridization between $\mathrm{Ti}^{-}$ $3 \mathrm{~d}$ orbitals and C-2p orbitals, and to its carbon vacancies. Our results show that the higher content of the light element is not the critical factor for high hardness, and carbon vacancies are also an effective way to enhance hardness.

\section{EXPERIMENT}

Cubic polycrystalline $\mathrm{TiC}_{1-\mathrm{x}}$ was prepared from titanium metal powder (99.9\% in purity) and graphite powder $(99.99 \%$ in purity). The raw powders were mixed in a ratio of $\mathrm{Ti}: \mathrm{C}=1: 1$ $\mathrm{x}$, where $x=0.5,0.4,0.3,0.2,0.1,0$. An agate mortar and a pestle were used to obtain homogenous mixtures. For each mixture, the raw material was cold-pressed into cylindrical pellets ( $4 \mathrm{~mm}$ in diameter, $2.5 \mathrm{~mm}$ in height) and sealed in a hexagonal$\mathrm{BN}$ capsule used to prevent the sample from reacting with the graphite heater. The high-pressure and high-temperature synthesis experiments were conducted on an SPD $6 \times 600 \mathrm{~T}$ cubic multi-anvil press. The powder mixture was heated to the target temperature $(1,400-2,300 \mathrm{~K})$ and soaked for $15 \mathrm{~min}$ at an elevated pressure of $5.0 \mathrm{GPa}$. Subsequently, the sample was cooled to room temperature $(300 \mathrm{~K})$ by turning off the power supply before decompression.

The as-synthesized polycrystalline samples were crushed to powder and their structure was characterized by X-ray diffraction with monochromatic $\mathrm{Cu} \mathrm{K}_{\alpha}$ radiation (1.5418 $\AA$ wavelength, with voltage and current of $40 \mathrm{KV}$ and $30 \mathrm{~mA}$, respectively). The microscope morphology and the element composition of the as-obtained polycrystalline specimens were investigated by using a scanning electron microscope (Magellan 400 SEM) equipped with an energy dispersive spectrum (EDS). The crystal structure of $\mathrm{TiC}_{1-\mathrm{x}}$ was investigated by selected area electron diffraction (SAED) and high-resolution transition electron microscopy (HRTEM) in a transmission electron microscope, JEM-2200FS. The as-prepared polycrystalline specimens were first polished by using silicon carbide paper and then diamond powder to achieve a mirror surface. Then, to obtain the average Vickers hardness, at least five indentations were made on the polished sample surface at different applied loads with a dwell time of $15 \mathrm{~s}$. Prior to the measurement of the binding energy of titanium and carbon, the surfaces of the polycrystalline specimens were bombarded by $\mathrm{Ar}^{+}$ions for $600 \mathrm{~s}$. XPS measurements were conducted with an X-ray source equipped with a $\mathrm{Mg}$ anode $(\mathrm{h} v=1253.6 \mathrm{eV})$ at a base pressure of $10^{-10}$ mbar achieved using an ultrahigh vacuum (UHV) chamber.

To investigate the electronic structure of TiC, we performed first-principles calculation using a Vienna ab initio Simulation Package (VASP) [20]. The Perdew-Burk-Ernzerhof (PBE) exchange-correlation was used in the generalized gradient approximation GGA [21]. An energy cutoff of $500.0 \mathrm{eV}$ and a dense Monkhorst-pack grid were selected to guarantee that the energy difference would be $<1.0 \mathrm{meV} /$ atom. The mechanical properties were calculated based on CASTEP code with a $2 \times 2 \times 2$ supercell, in which $300.0 \mathrm{eV}$ and $2 \pi \times$ $0.03 \AA^{-1}$ were chosen as the energy cutoff and k-mesh value, respectively $[20,22,23]$.

\section{RESULTS AND DISCUSSION}

The polycrystalline specimens of $\mathrm{TiC}_{1-\mathrm{x}}$ were synthesized at the high pressure of 5.0 $\mathrm{GPa}$ and a temperature between 1,900 and $2,300 \mathrm{~K}$, with a duration time of $15 \mathrm{~min}$. Titanium metal powders start to react with graphite powder at temperatures above $1,600 \mathrm{~K}$, and pure cubic polycrystalline $\mathrm{TiC}_{1-\mathrm{x}}$ was synthesized at the temperature above $1,900 \mathrm{~K}$. The room-temperature $\mathrm{X}$ ray diffraction profiles of different quenched polycrystalline specimens of $\mathrm{TiC}_{1-\mathrm{x}}$ are shown in Figure 1a. The major peaks of $\mathrm{TiC}_{0.7} \mathrm{X}$-ray diffractions are well-characterized by the cubic rock-salt structure possessing parameters $a=b=c=4.3254$ $\AA$, which is in fairly good agreement with the previous report [18]. X-ray diffraction (XRD) shows a tiny peak at $26.8^{\circ}$ 
which is corresponding to the graphite phase, and this can be detected in the expanded XRD profiles. The tiny peak of graphite suggests that a certain amount of graphite is excluded from $\mathrm{TiC}$ lattice. Therefore, it is likely that carbon vacancies are present in the $\mathrm{TiC}_{1-\mathrm{x}}$ structure. Because the tiny graphite peaks did not provide detailed structural information for $\mathrm{TiC}_{1-\mathrm{x}}$, we could not confirm the relative number of carbon atoms that were excluded from the $\mathrm{TiC}$ cubic structure. To investigate carbon atom exclusion, we compared specimens with different starting material ratios of Ti:C $=1: 1-x$. Figure $\mathbf{1 b}$ shows the diffraction peaks change with the increasing of $x$. They first move to the left, which suggests that the lattice parameters become smaller. When $x$ reaches 0.3 , it is difficult to see any graphite diffraction peaks, and we tentatively determine the amount of carbon vacancy to be $\sim 30 \%$. Though the raw material mixes were stoichiometric and the compounds were synthesized under high pressure and high temperature, the appearance of graphite diffraction peaks indicates that the carbon vacancies are extremely robust. When $x$ is larger than $0.5, \mathrm{X}$-ray diffraction shows additional peaks of other-phase. The appearance of other phases probably indicates that the $\mathrm{x}$ limit for synthesizing $\mathrm{TiC}_{1-\mathrm{x}}$ is 0.5 .

Further SEM observations revealed that the synthesized $\mathrm{TiC}_{0.7}$ specimens were free of visible micro-cracks and pores, with a high relative density of $\sim 97 \%$. Figures 1 c,d shows typical field scanning electron microscope images of fracture section at different arbitrary locations. From Figure 1c, we can see that the as-synthesized specimens exhibit highly crystalline grains. The size of the grain is determined to be $20-40 \mu \mathrm{m}$. Besides, laminar morphology also can be seen in the Figure 1d, which originates from the crack of the specimen. The elemental composition and ratio in the synthesized specimen were confirmed by EDS to be about $\mathrm{TiC}_{0.7}$, suggesting that the synthesized specimen is non-stoichiometric. This is in agreement with the $a b$ initio composition of this specimen. The SAED patterns revealed that the diffraction spots are in good order and determine it to be a cubic structure, as shown in Figure 1e. It is difficult to detect any satellite spots around the main spots, thereby ruling out the possibility that carbon vacancies form a superstructure in the host lattice [24]. The specimen was also studied using high-resolution transmission electron microscope (HRTEM). As can be seen from Figure 1f, the inter-planar spacing was found to be $2.58 \AA$, which corresponds to the (111) facet of TiC. Although there is $30 \%$ carbon vacancy, the specimen still exhibits a highly crystalline nature, as can be seen from the HRTEM images.

Considering that the number of carbon vacancies does not vary significantly with variations in starting compositions when $\mathrm{x}$ is larger than 0.3 , we concluded that the influence of residual graphite precursor on the hardness can be ignored for $\mathrm{TiC}_{0.7}$. If the specimen exhibits high hardness, there will be a strong orbital hybridization between the transition metal-d orbital and the C-2p orbital, which has been proposed to be beneficial for high hardness. Measurement of the hardness of $\mathrm{TiC}_{0.7}$ specimen was conducted on the polished mirror surface using the Vickers hardness scale with a pyramid diamond indenter. The loading force exerted on the specimens ranged from 25 to $1,000 \mathrm{~g}$ until a converged Vickers hardness was obtained. The micro Vickers hardness value was confirmed according to Equation (1):

$$
H_{v}=1.8544 \frac{p}{d^{2}}
$$

in which $d$ is the length of the two diagonals of the indentations and $P$ is the applied force with a duration time of $15 \mathrm{~s}$. At least five indentations were made on the surface of the sample to acquire accurate hardness data. The average Vickers hardness value of a polycrystalline $\mathrm{TiC}_{0.7}$ specimen at different loading forces is given in the inset of Figure 2A. The hardness decreases with the increase in loading force. With increasing of loading force, Vickers hardness gradually converged to an asymptotic Vickers hardness value of 27.1 GPa. Compared with the previous results of $24.7 \mathrm{GPa}$, the measured asymptotic Vickers hardness of samples fabricated under HPHT conditions is slightly higher. The higher Vickers hardness specimen obtained by HPHT synthesis is not a surprise because the measured hardness is closely related to the quality of the sample. Because the pores filling effect will contribute to the size of hardness indentation, a higher relative density of sample suggests that the obtained hardness value will be closer to the value of a certain material. The HPHT method is demonstrated to be effective in the preparation of high-relative-density specimens.

Figure 2B shows the Vickers hardness values of different transition metal borides with high boron concentration, widelyused transition metal carbides, and our titanium carbide specimen. We can see that the asymptotic Vickers hardness is even greater than that of $\mathrm{ReB}_{2}, \mathrm{WB}_{3}, \mathrm{ReB}_{2}, \mathrm{MnB}_{4}, \mathrm{ZrB}_{12}$, and even $\mathrm{FeB}_{4}$, which was previously believed to be superhard $[2,11,12,25]$. When it is compared with other widely used transition metal carbides (WC, WC-8\%Co, TaC, and $\mathrm{Mo}_{3} \mathrm{C}_{2}$ ), $\mathrm{TiC}_{\mathrm{x}}$ exhibit higher hardness. The low light element content also induces higher hardness in $\mathrm{TiC}$ [26-29]. This fact indicates that high light-element content or higher-dimensional light-element frameworks may not be the critical criterion for designing superhard materials.

Bulk modulus (B) denotes the ability of a material to resist volume change under hydrostatic pressure. Superhard materials usually possess a high bulk modulus value, though the inverse may not always be true [30]. The unit cell of TiC crystallizes in a cubic structure with lattice parameter $a=4.32 \AA$, yielding a unit cell volume of $80.62 \AA^{3}$. Thus, the molecular volume is 20.16 $\AA^{3}$. The valence electron shell composition of Ti is $3 \mathrm{~d}^{2} 4 \mathrm{~s}^{2}$ or $Z=$ 4 , and that of $C$ is $2 s^{2} \mathrm{p}^{2}$ with $Z$ of 4 . Thus, the total number of valence electrons in one unit cell is eight and the valence electron density (VED) is 0.4 electrons $/ \AA^{3}$. Diamond has the reported highest bulk modulus of $442.0 \mathrm{GPa}$ and a VED of 0.7 electron $/ \AA^{3}$, and the most in-compressible metal, Os, has a bulk modulus of $411.0 \mathrm{GPa}$ and a VED of 0.57 electrons/ $\AA^{3}$ [8]. Relatively low VED cannot guarantee an exceptionally high bulk modulus structure to TiC. Consequently, the calculated bulk modulus of $\mathrm{TiC}$ from first-principles calculation is only $252.4 \mathrm{GPa}$, which is far lower than that of diamond, Os, and the osmium di-borides. However, according to recent reports of S. H. Jhi and Y. C. Liang, a valence electron number of eight will result in the most 

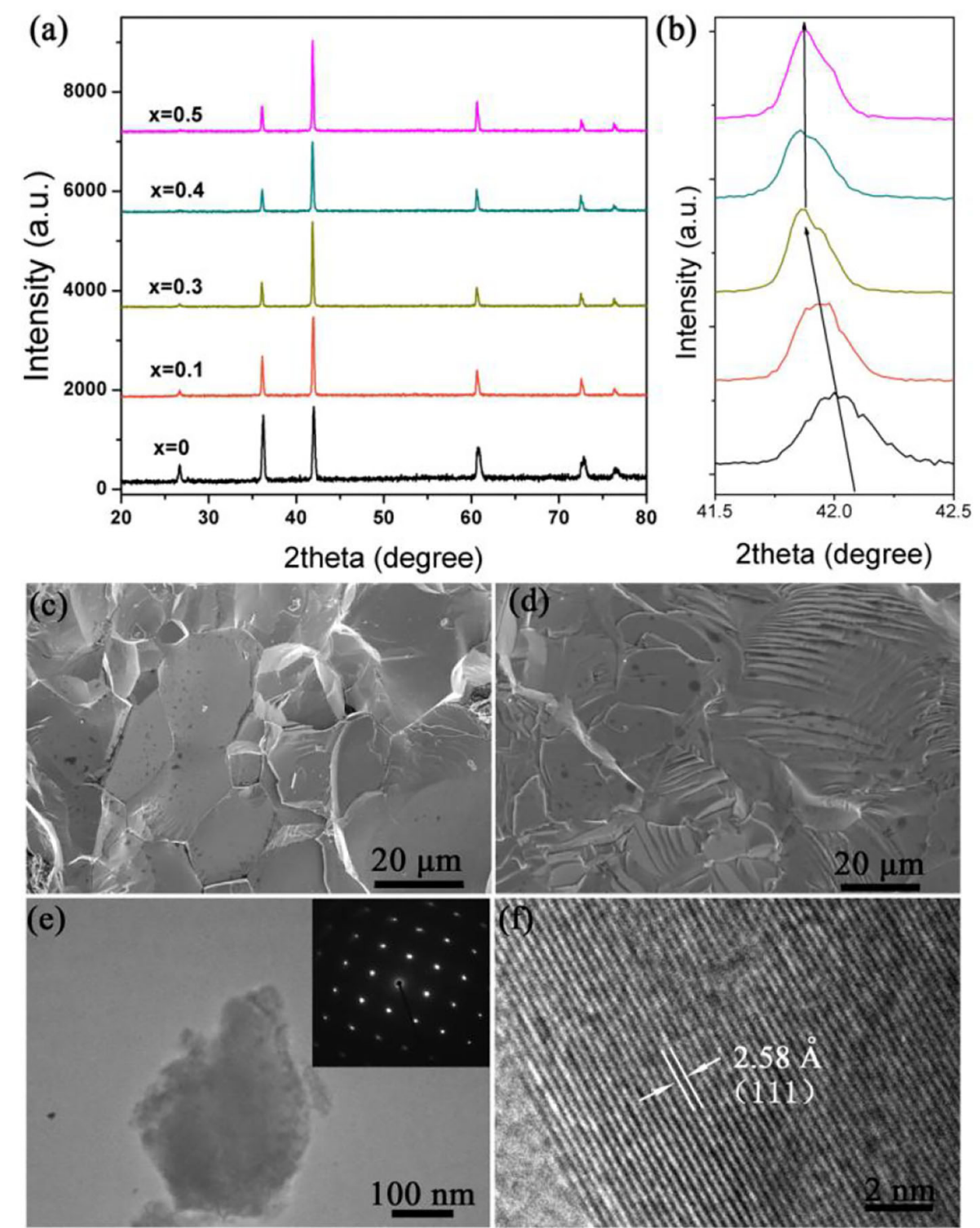

FIGURE 1 | (a) X-ray powder diffraction profiles of $\mathrm{TiC}_{1-x}$ specimens $(x=0,0.1,0.2,0.3,0.4,0.5)$ synthesized at 2,000 K, 5.0 GPa, 15 min. (b) Enlarged X-ray diffraction peak position of (200) plane with decreasing carbon content. (c,d) Typical field scanning electron microscope (SEM) photographs of TiC 0.7 at different regions. (e) Bright field images of the synthesized specimen. The selected electron area diffraction patterns are given in the inset. (f) High-resolution TEM image of the obtained specimen.

reasonable electronic structure, and thus higher hardness [3134]. The valence electron of $\mathrm{TiC}$ is about eight electrons, and this may be one of the reasons for its exceptionally high hardness.

Recent investigations of the microscopic level have revealed that hardness of a solid is dependent on the bond density, bond length, bond strength, and bond electro-negativity. According to previous results, the predominant bond in the rock-salt structure of transition metal mono-carbides is TM-C bonds formed by the hybridization between transition-metal-d orbitals and C-2p orbitals. It is necessary to study this bond characteristic and the electronic configurations of $\mathrm{TiC}$ because the feature of chemical bonds is critical for the mechanical properties. One of the most promising means for doing this would be through the combination of the interrelationships between the first-principles calculation and the binding-energy measurements. Figure 3B shows the XPS spectrum of C-1s with binding energy from 283.0 to $292.0 \mathrm{eV}$. As can be seen from the spectrum, there are two peaks at the binding energy of 284.7 and $287.5 \mathrm{eV}$. The binding energy of graphite 1 s peak usually locates at $284.5 \mathrm{eV}$. Therefore, the peak at the binding energy of $284.7 \mathrm{eV}$ belongs to the residual graphite extracted from the $\mathrm{TiC}$ host matrix. The appearance of the graphite peak confirms that there are some carbon vacancies 

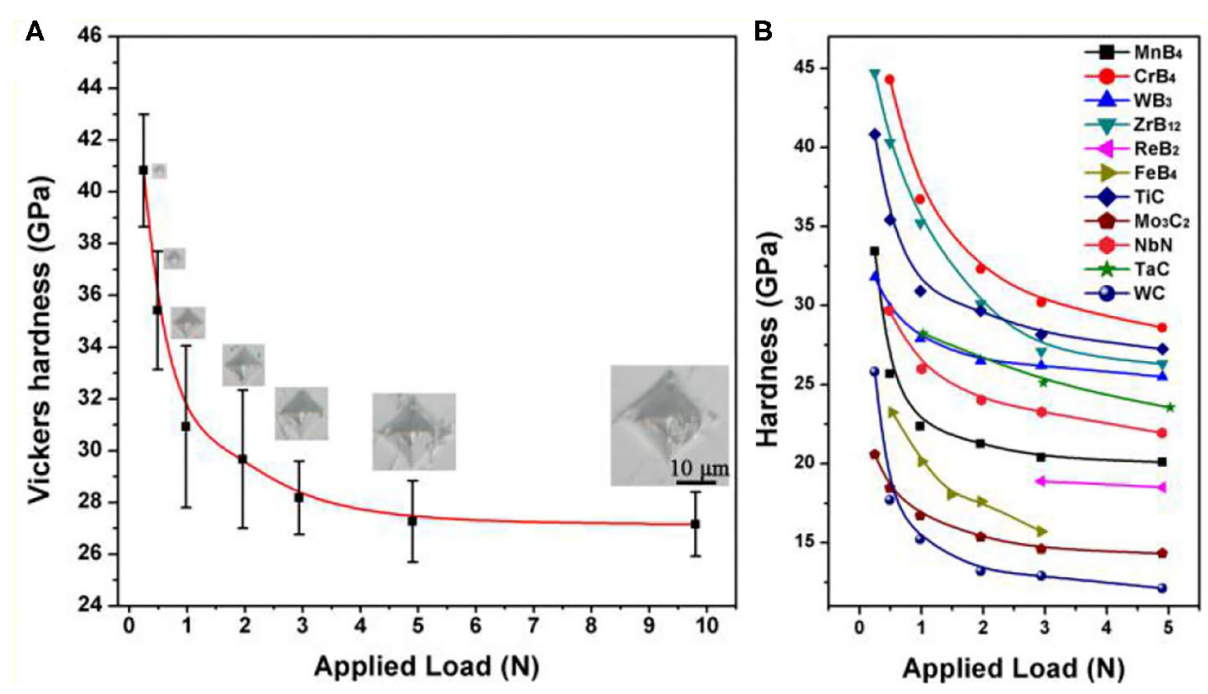

FIGURE 2 | (A) Vickers hardness value obtained as a function of the applied load of TiC 0.7 . The corresponding indentation images at different applied loads are presented above each data point. (B) Vickers hardness values of high boron concentration transition metal borides and titanium carbide.

in the synthesized polycrystalline specimens. Another peak at the higher binding energy of $287.5 \mathrm{eV}$ can be assigned to TiC. The fact that binding energy is higher than that of graphite suggests an electron transfer from titanium to carbon atoms. Figure 3A presents the Ti-2 $\mathrm{p}^{3 / 2}$ and $2 \mathrm{p}^{1 / 2}$ spectra from the binding energy of 455 to $468 \mathrm{eV}$. There are two pairs of binding energy peaks in the Ti 2p XPS spectra. The two main peaks at 457.7 and $463.7 \mathrm{eV}$ are attributed to the highly crystalline $\mathrm{TiC}$, and the pair of small peaks at 458.7 and $464.6 \mathrm{eV}$ is assigned to the defect structure. Comparing the peak area of two pair peaks of $\mathrm{TiC}$, the carbon vacancies, $x$ can be confirmed to be about 0.37 to 0.38 , which is approximately the same as the previous $\mathrm{EDS}$ result of $\mathrm{TiC}_{0.7}$. The $\mathrm{C}$-1s binding energy of $\mathrm{TiC}$ is higher than that of graphite, which means that some electrons are transformed from titanium atoms to carbon atoms. The charge transform between carbon and titanium suggests that there is some ionic characteristic in the Ti-C bond.

The hardness of TiC was also calculated with Chen's model with the following Equation,

$$
H_{v}=2\left(k^{2} G\right)^{0.585}-3
$$

[35]. The calculated bulk modulus and shear modulus are 252.4 and $176.1 \mathrm{GPa}$, respectively. Based on the above theoretical modulus, the simulated Vickers hardness is about 24.0 GPa, which agrees with the experimental asymptotic hardness value. To further investigate the electronic properties and the bond characteristic of TiC, first-principles calculations were performed by using the CASTEP code.According to the crystal structure and the electron location function (ELF) of rock salt TiC, as shown in Figure 4A,B, only one type of Ti-C bond exists in the $\mathrm{TiC}$ structure. Figure $4 \mathrm{C}$ presents the total and partial density of states of TiC. One can observe a peculiar bonding configuration from the density of state, indicating that there is high degree of matching between the Ti-3d orbitals and C- $2 \mathrm{p}$

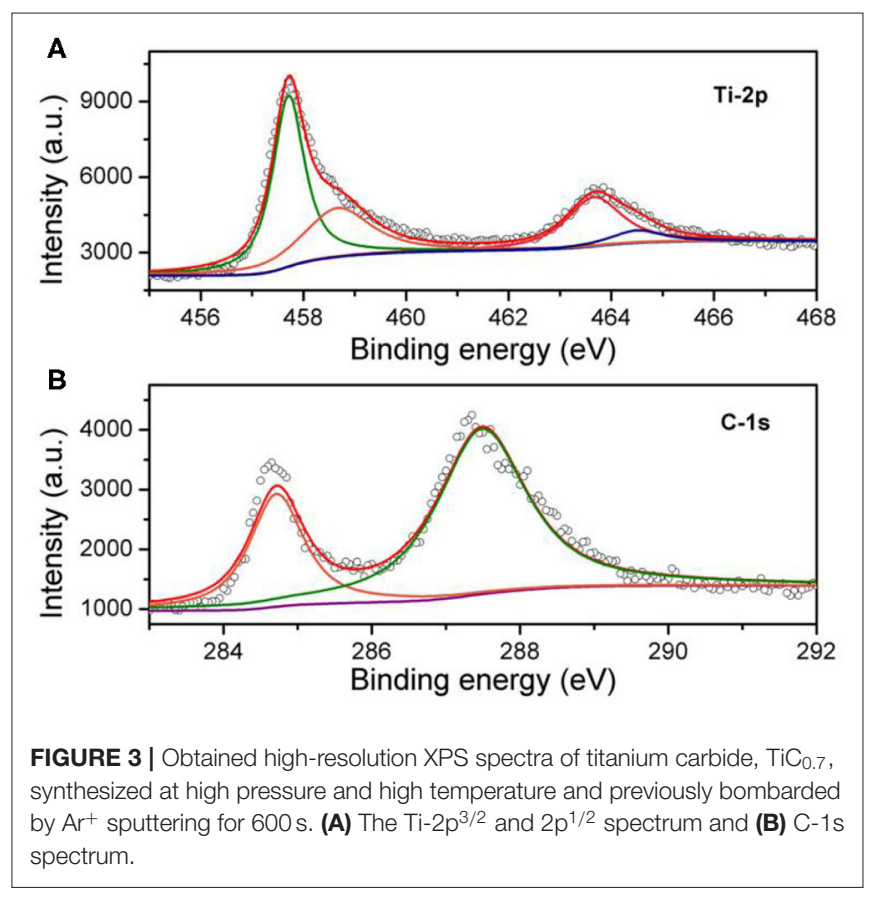

orbitals from -3 to $-1 \mathrm{eV}$. The match of the partial density of states (PDOS) suggests that the Ti-C bond in the crystal lattice is also of some strong covalent feature. The non-zero electron density of state at the Fermi level indicates a metallic feature of the TiC crystal structure, which is consistent with the electrical conductive resistivity of $3.56 \times 10^{-7} \Omega \cdot \mathrm{m}$. To further quantitatively describe the bonding nature of $\mathrm{TiC}$, we conducted the Mulliken population analysis. A high overlap between the electron configuration or the higher Mulliken population corresponds to a strong covalent bond, whereas a value close 


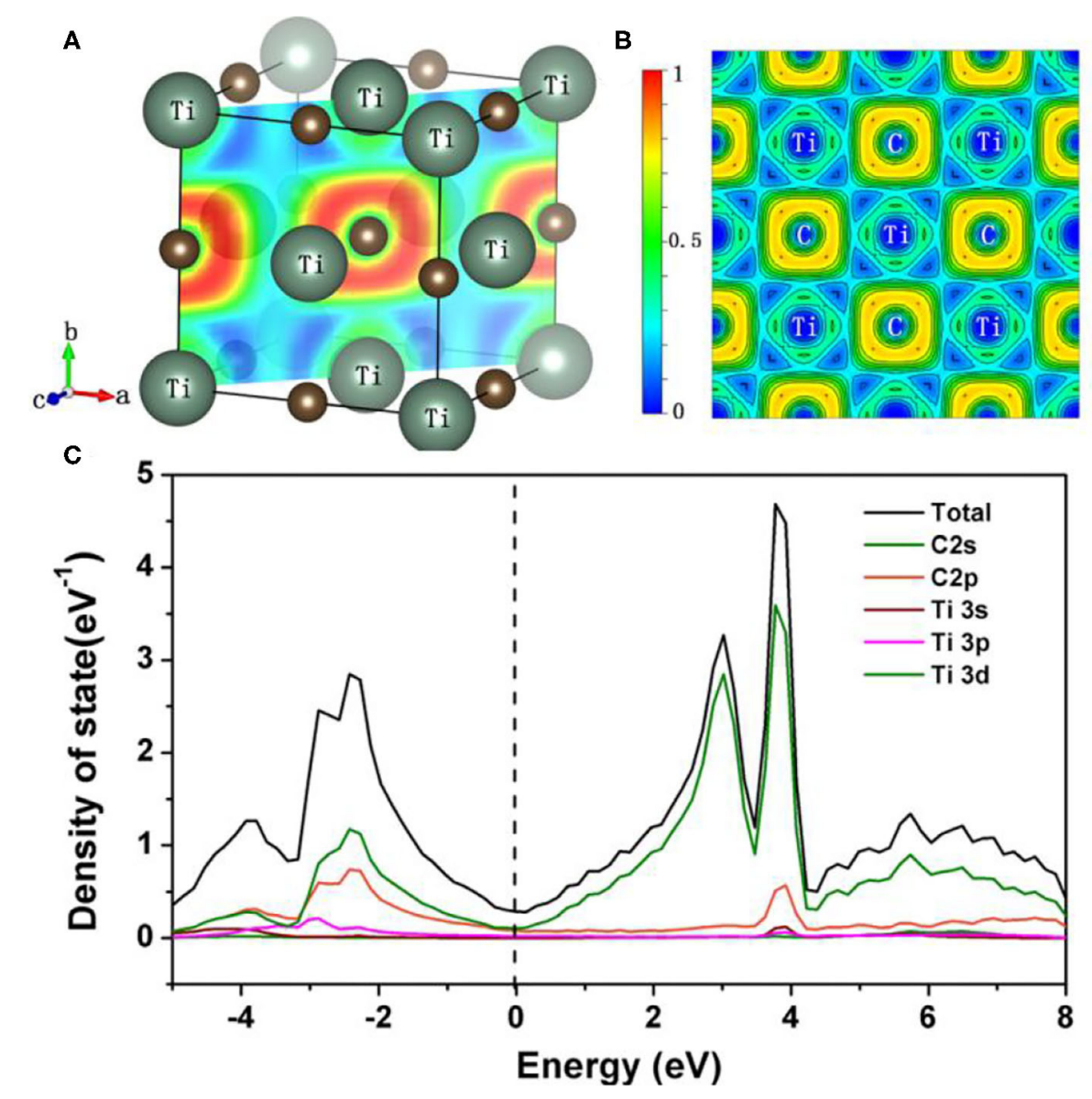

FIGURE 4 | (A,B) ELF and CDD slices of (110) plane of TiC. (C) Calculated density of states of TiC. The Fermi energy level ( $\left.E_{F}\right)$ is shown by a vertical dashed line.

to zero indicates weak interactions between two atoms. The calculation results confirmed our previous assumption on the bonding. Compared with the Mulliken population of $\mathrm{C}-\mathrm{C}$ bonds in diamond, $P v=0.75, \mathrm{Re}-\mathrm{B}$ in $\mathrm{ReB}_{2}, P v=0.73$ and $\mathrm{B} 1-\mathrm{B} 1$ in $\mathrm{WB}_{4}$, one can draw the conclusion that the $\mathrm{Ti}$ C bond, $P v=0.81$, has the characteristic of covalent bond [36]. Moreover, the calculation of the Mulliken population also reveals that charge transfer definitely occurred between $\mathrm{Ti}$ and $\mathrm{C}$ atoms in the Ti-C bond. The atomic average Mulliken charges are 0.84 and -0.84 for $\mathrm{C}$ and $\mathrm{Ti}$. The electron transfer from titanium atoms to carbon atoms is inconsistent with the conclusion of XPS. The Mulliken population analysis results also confirmed that the Ti-C bond possesses ionic characteristics at the same time. This is consistent with the results of XPS. Thus, the bonds of $\mathrm{TiC}$ have a combination of covalent metallic and ionic characteristics. However, ionic and metallic bonds are detrimental to hardness according to previous reports. Consequently, it is advisable to keep away from metallic and ionic bonds in transition metal carbide when synthesizing superhard materials. However, compared with the $\mathrm{TiC}$ specimens fabricated by other methods, the synthesized $\mathrm{TiC}_{1-\mathrm{x}}$ specimen exhibited higher hardness value. This tentatively comes from extrinsic factor. Because defects act as pinning centers, defects can hinder the motion of dislocation and thus enhance the mechanical strength. In addition, the emergence of carbon vacancies alters the electronic number in the crystal structure and result in the optimized electronic structure $[33,34]$. Consequently, $\mathrm{TiC}_{1-\mathrm{x}}$ specimens exhibit extraordinary higher hardness than other fabrication methods.

\section{CONCLUSIONS}

In summary, carbon-deficient $\mathrm{TiC}_{1-\mathrm{x}}$ specimens were synthesized under conditions of high temperature and high pressure. The synthesized specimens exhibit relatively high hardness, higher than many high-boron-concentration compounds. Based on the result of a first-principles calculation, we conclude that the higher hardness of $\mathrm{TiC}$ can be attributed to the strong $\mathrm{Ti}-\mathrm{C}$ bonds and the optimized electronic structure. In addition, the carbon vacancies also play an important role in enhancing hardness by extrinsic harden method. Although the carbon vacancy is good for high hardness, carbon vacancies in these specimens tend to be robust even under HPHT synthesis conditions. Further investigation of critical synthesis conditions may be required to adjust the carbon 
vacancies issue and achieve the higher hardness expected for stoichiometric TiC.

\section{DATA AVAILABILITY STATEMENT}

The raw data supporting the conclusions of this article will be made available by the authors, without undue reservation.

\section{AUTHOR CONTRIBUTIONS}

LC and ZY contributed the conception and design of the study. $\mathrm{ZY}$ organized the literature. HL and SM performed the design of

\section{REFERENCES}

1. Knappschneider A, Litterscheid C, Kurzman J, Seshadri R, Albert B. Crystal structure refinement and bonding patterns of $\mathrm{crB}_{4}$ : a Boron-Rich boride with a framework of tetrahedrally coordinated B atoms. Inorg Chem. (2011) 50:10540-2. doi: 10.1021/ic2018083

2. Qin J, He D, Wang J, Fang L, Lei L, Li Y, et al. Is rhenium diboride a superhard material? Adv Mater. (2008) 20:4780-3. doi: 10.1002/adma.200801471

3. Kaner RB, Gilman JJ, Tolbert SH. Designing superhard materials. Science. (2005) 308:1268-9. doi: 10.1126/science.1109830

4. Gu Q, Krauss G, Steurer W. Transition metal borides: superhard versus ultra-incompressible. Adv Mater. (2008) 20:3620-6. doi: 10.1002/adma.2007 03025

5. Mohammadi R, Lech AT, Xie M, Weaver BE, Yeung MT, Tolbert SH, et al. Tungsten tetraboride, an inexpensive superhard material. PNAS. (2011) 108:10958-62. doi: 10.1073/pnas.1102636108

6. Ma S, Bao K, Tao Q, Zhu P, Ma T, Liu B, et al. Manganese mono-boride, an inexpensive room temperature ferromagnetic hard material. Sci Rep. (2017) 7:43759. doi: 10.1038/srep43759

7. Li H, Wen P, Li Q, Dun C, Xing J, Lu C, et al. Earth-Abundant iron diboride $\left(\mathrm{FeB}_{2}\right)$ nanoparticles as highly active bifunctional electrocatalysts for overall water splitting. Adv Energy Mater. (2017) 7:1700513. doi: 10.1002/aenm.201700513

8. Gilman JJ, Cumberland RW, Kaner RB. Design of hard crystals. Int J Refractory Metals Hard Mater. (2006) 24:15. doi: 10.1016/j.ijrmhm.2005.05.015

9. Guo X, Li L, Liu Z, Yu D, He J, Liu R, et al.-Wang T. Hardness of covalent compounds: roles of metallic component and D valence electrons. J Appl Phys. (2008) 104:023503. doi: 10.1063/1.2956594

10. Wang Q, He J, Hu W, Zhao Z, Zhang C, Luo $K$, et al. Is orthorhombic iron tetraboride superhard? J Materiom. (2015) 1:45-51. doi: 10.1016/j.jmat.2015.03.004

11. Tao Q, Zheng D, Zhao X, Chen Y, Zhu P. Exploring hardness and the distorted sp2 hybridization of $\mathrm{b}-\mathrm{B}$ bonds in $\mathrm{wB}_{3}$. Chem Mater. (2014) 26:5297-302. doi: 10.1021/cm5021806

12. Ma S, Bao K, Tao Q, Huang Y, Xu C, Li L, et al. Investigation the origin and mechanical properties of unusual rigid diamond-like net analogues in manganese tetraboride. Int J Refractory Metals Hard Mater. (2019) 85:104845. doi: 10.1016/j.ijrmhm.2018.12.010

13. Tang H, Gao X, Zhang J, Gao B, Gou H. Boron-rich molybdenum boride with unusual short-range vacancy ordering, anisotropic hardness, and superconductivity. Chem Mater. (2019) 32:45967. doi: 10.1021/acs.chemmater.9b04052

14. Ma T, Li H, Zheng X, Wang S, Wang X, Zhao H. Ultrastrong boron frameworks in $\mathrm{zrB}_{12}$ : a Highway for electron conducting. Adv Mater. (2016) 29:1604003. doi: 10.1002/adma.201604003

15. Wang C, Tao Q, Ma S, Cui T, Wang X, Dong S, et al. $\mathrm{WB}_{2}$ : not a superhard material for strong polarization character of interlayer $\mathrm{w}-\mathrm{B}$ bonding. Phys Chem Chem Phys. (2017) 19:8919-24. doi: 10.1039/C6CP04287B figures and wrote the first draft of the manuscript. All authors contributed to manuscript revision, read, and approved the submitted version.

\section{FUNDING}

This research was supported by Guangxi Natural Science Foundation under Grant No. 2018GXNSFBA050034 and 2019GXNSFBA185001, the Young and middle-aged teachers basic ability improvement project of Guangxi Department of education, No. 2020KY04022.

16. Yeung MT, Akopov G, C.-Lin W, King DJ, Li RL, Sobell ZC, et al. Superhard $\mathrm{w}_{0.5} \mathrm{Ta}_{0.5} \mathrm{~B}$ nanowires prepared at ambient pressure. Appl Phys Lett. (2016) 109:203107. doi: 10.1063/1.4967447

17. Li Q, Zhou D, Zheng W, Ma Y, Chen C. Anomalous stress response of ultrahard $\mathrm{wBn}$ compounds. Phys Rev Lett. (2015) 115:185502. doi: 10.1103/PhysRevLett.115.185502

18. Raghuram Bunshah CARF. The effect of substrate temperature on the structure of titanium carbide deposited by activated reactive evaporation. $J$ Vacuum Sci Technol. (1972) 9:1389-94. doi: 10.1116/1.1317046

19. Kumashiro $\mathrm{Y}$, Itoh A, Kinoshita T, Sobajima M. The micro-Vickers hardness of $\mathrm{TiC}$ single crystals up to $1500^{\circ} \mathrm{C}$. J Mater. (1977) 12:595601. doi: 10.1007/BF00540285

20. Kresse G. Efficient iterative schemes for ab initio total-energy calculations using a plane-wave basis set. Phys Rev B. (1996) 54:11169-86. doi: 10.1103/PhysRevB.54.11169

21. Perdew JP, Burke K, Ernzerhof M. Generalized gradient approximation made simple. Phys Rev Lett. (1996) 77:3865-8. doi: 10.1103/PhysRevLett.77.3865

22. Segall MD, Lindan PJD, Probert MJ, Pickard CJ, Hasnip PJ, Clark SJ, et al. First-principles simulation: ideas, illustrations and the CASTEP code. J Phys Condens Matter. (2002) 14:2717-44. doi: 10.1088/0953-8984/14/11/301

23. Segall MD, Shah R, Pickard CJ, Payne MC. Population analysis of planewave electronic structure calculations of bulk materials. Phys Rev B. (1996) 54:16317-20. doi: 10.1103/PhysRevB.54.16317

24. Sathish CI, Guo Y, Wang X, Tsujimoto Y, Li J, Zhang S, et al. Superconducting and structural properties of d-MoC0.681 cubic molybdenum carbide phase. $J$ Solid State Chem. (2012) 196:579-85. doi: 10.1016/j.jssc.2012.07.037

25. Wang S, Yu X, Zhang J, Zhang Y, Wang L, Leinenweber K, et al. Crystal structures, elastic properties, and hardness of high pressure synthesized $\mathrm{crB}_{2}$ and $\mathrm{crB}_{4} . \quad J$ Superhard Mater. (2014) 36:279-87. doi: 10.3103/S1063457614040066

26. Chen Y, He D, Qin J, Kou Z, Bi Y. Ultrasonic and hardness measurements for ultrahigh pressure prepared wB ceramics. Int J Refractory Metals Hard Mater. (2011) 29:329-31. doi: 10.1016/j.ijrmhm.2010.12.006

27. Sun W, Kuang X, Liang H, Xia X, Zhang Z, Lu C, et al. Mechanical properties of tantalum carbide from high-pressure/high-temperature synthesis and first principles calculations. Phys Chem Chem Phys. (2020) 22:1-6. doi: 10.1039/C9CP06819H

28. Ge Y, Ma S, Kuo B, Tao Q, Zhao X, Feng X, et al. Superconductivity with high hardness in $\mathrm{Mo}_{3} \mathrm{C}_{2}$. Inorg Chem Front. (2019) 6:12828. doi: 10.1039/C9QI00182D

29. Zou Y, Wang X, Chen T, Li X, Qi X, Welch D, et al. Hexagonal-structured $\varepsilon-\mathrm{NbN}$ : ultra-incompressibility, high shear rigidity, and a possible hard superconducting material. Sci Rep. (2015) 5:10811. doi: 10.1038/srep10811

30. Cumberland RW, Weinberger MB, Gilman JJ, Clark SM, Tolbert SH, Kaner RB. Osmium diboride, an ultra-Incompressible, hard material. J Am Chem Soc. (2005) 127:7264-5. doi: 10.1021/ja043806y

31. Liang Y, Gao Z, Qin P, Gao L, Tang C. The mechanism of anomalous hardening in transition-metal monoborides. Nanoscale. (2017) 9:91128. doi: 10.1039/C7NR02377D 
32. Liang Y, Qin P, Jiang H, Zhang L, Zhang J, Tang C. Designing superhard metals: the case of low borides. AIP Adv. (2018) 8:045305. doi: 10.1063/1.50 23830

33. Jhi SH, Ihm J, Louie SG, Cohen ML. Electronic mechanism of hardness enhancement in transition-metal carbonitrides. Nature. (1999) 399:13234. doi: $10.1038 / 20148$

34. Jhi SH, Louie SG, Cohen ML, Ihm J. Vacancy hardening and softening in transition metal carbides and nitrides. Phys Rev Lett. (2001) 86:3348-51. doi: 10.1103/PhysRevLett. 86.3348

35. Chen X-Q, Niu H, Li D, Li Y. Modeling hardness of polycrystalline materials and bulk metallic glasses. Intermetallics. (2011) 19:1275-81. doi: 10.1016/j.intermet.2011. 03.026
36. Wang M, Li Y, Cui T, Ma Y, Zou G. Origin of hardness in $\mathrm{wB}_{4}$ and its implications for $\mathrm{reB}_{4}, \mathrm{taB}_{4}, \mathrm{moB}_{4}, \mathrm{tcB}_{4}$, and $\mathrm{osB}_{4}$. Appl Phys Lett. (2008) 93:101905. doi: 10.1063/1.2977760

Conflict of Interest: The authors declare that the research was conducted in the absence of any commercial or financial relationships that could be construed as a potential conflict of interest.

Copyright (C) $2020 \mathrm{Li}, \mathrm{Ma}$, Chen and Yu. This is an open-access article distributed under the terms of the Creative Commons Attribution License (CC BY). The use, distribution or reproduction in other forums is permitted, provided the original author(s) and the copyright owner(s) are credited and that the original publication in this journal is cited, in accordance with accepted academic practice. No use, distribution or reproduction is permitted which does not comply with these terms. 